\title{
La Política de empleo como garantía del «trabajo decente». Retos para Cuba ante los sujetos económicos no estatales
}

The Employment Policy as a guarantee of "decent work". Challenges for Cuba before non-state economic subjects.

A Política de Emprego como garantia de "trabalbo decente". Desafios para Cuba diante de temas econômicos não estatais.

La politique de l'emploi en tant que garantie de "travail décent". Défis pour Cuba devant des sujets économiques non étatiques 就业政策是“体面劳动”的保证。在非国家经济主体面前对古 巴的挑战

Reynaldo Lam Peña ${ }^{1}$ Universidad de La Habana

Revista Derechos en Acción ISSN 2525-1678/ e-ISSN 2525-1686

Año 4/Nº 11 Otoño 2019 (21 marzo a 21 junio), 256-276

DOI: https://doi.org/10.24215/25251678e275

ORCID: https://orcid.org/0000-0003-0629-4612

Recibido 17/02/2019

Aprobado: 04/05/2019

Resumen: El «trabajo decente» es un concepto de contenido social y ético enunciado por la Organización Internacional del Trabajo a finales del siglo pasado. La doctrina le otorga contenido a este enunciado a través de 4 elementos: el acceso al empleo, los derechos laborales, la protección social y el diálogo social. El presente ensayo realiza un análisis del primero de estos elementos a través de su materialización en la Política de Empleo, entendida como un complejo integrativo y

1 Abogado, adjunto cátedra III de Historia Constitucional, Facultad de Ciencias Jurídicas y Sociales, UNLP. 
transversal de políticas públicas esbozadas por órganos de la Administración Central del Estado con la finalidad de garantizar el fomento del empleo, el pleno acceso y la igualdad en el disfrute del derecho al trabajo como postulado constitucional. En Cuba, la Política de empleo expone como sus principios fundamentales la garantía, libertad y pleno empleo para todas las personas con capacidad de trabajar. No obstante, ante los recientes cambios legislativos y el reconocimiento jurídico de nuevos sujetos económicos, el Estado cubano posee el reto de incorporar a estos sujetos a las estrategias de políticas de empleo que busquen la consecución del acceso al empleo como uno de los elementos que garantiza el "trabajo decente» en Cuba y el disfrute del «empleo digno» tal como establece uno de los postulados constitucionales de la norma patria vigente.

Palabras claves: trabajo decente- Política de empleo- derecho al trabajosujetos económicos

Abstract: "Decent work" is a concept of social and ethical content enunciated by the International Labor Organization at the end of the last century. The doctrine gives content to this statement through 4 elements: access to employment, labor rights, social protection and social dialogue. This essay makes an analysis of the first of these elements through its materialization in the Employment Policy, understood as an integrative and transversal complex of public policies outlined by organs of the Central State Administration with the purpose of guaranteeing the promotion of employment, full access and equality in the enjoyment of the right to work as a constitutional postulate. In Cuba, the Employment Policy exposes as its fundamental principles the guarantee, freedom and full employment for all persons with the ability to work. However, given the recent legislative changes and the legal recognition of new economic subjects, the Cuban State has the challenge of incorporating these subjects into employment policy strategies that seek to achieve access to employment as one of the elements that guarantee "decent work" in Cuba and the enjoyment of "decent work" as established by one of the constitutional postulates of the current national norm.

Keywords: decent work - employment policy - right to work - economic subjects 
Resumo: 0 trabalho decente é um conceito de conteúdo social e ético enunciado pela Organização Internacional do Trabalho no final do século passado. A doutrina dá conteúdo a esta declaração através de 4 elementos: 0 acesso ao emprego, os direitos trabalhistas, a proteção social e o diálogo social. 0 presente ensaio faz uma análise do primeiro desses elementos através de sua materialização na Política de Emprego, entendida como um complexo integrador e transversal de políticas públicas delineadas por órgãos da Administração Central do Estado com o objetivo de garantir a promoção do emprego, o pleno acesso e a igualdade no gozo do direito ao trabalho como postulado constitucional. Em Cuba, a Política de emprego expõe como seus princípios fundamentais a garantia, liberdade e pleno emprego para todas as pessoas com capacidade de trabalhar. No entanto, antes as recentes mudanças legislativas e 0 reconhecimento legal de novos sujeitos econômicos, o Estado cubano tem 0 desafio de incorporar esses sujeitos nas estratégias de políticas de emprego que buscam o acesso ao emprego como um dos elementos que garantiza "trabalho decente" em Cuba e o gozo do "trabalho digno" tal como establece um dos postulados constitucionais da norma pátria vigente.

Palavras-chave: trabalho decente - Política de emprego - direito ao trabalho - sujeitos econômicos.

Résumé: Le "travail décent" est un concept de contenu social et éthique énoncé par l'Organisation internationale du Travail à la fin du siècle dernier. La doctrine donne contenu à cette déclaration à travers quatre éléments: accès à l'emploi, droits du travail, protection sociale et dialogue social. Le présent travail analyse le premier de ces éléments à travers sa concrétisation dans la politique de l'emploi, entendue comme un ensemble intégrateur et transversal de politiques publiques définies par les organes de l'administration centrale de l'État afin de garantir la promotion de l'emploi. , le plein accès et l'égalité dans la jouissance du droit au travail en tant que postulat constitutionnel. À Cuba, la politique de l'emploi énonce comme principes fondamentaux la garantie, la liberté et le plein emploi pour toutes les personnes ayant la capacité de travaiIler. Cependant, compte tenu des récents changements législatifs et de la reconnaissance juridique de nouveaux sujets économiques, l'État cubain a le défi d'intégrer ces sujets dans les stratégies de politique de l'emploi qui cherchent à assurer l'accès à "travail décent" à Cuba et la jouissance 
d'un "emploi décent" établi par l'un des postulats constitutionnels de la norme nationale en vigueur.

Mot-clés: Travail décent - Politique de l'emploi - droit au travail - sujets économiques.

摘要: “体面劳动”是社会和道德内容的概念。该学说通过4个要素为 这一陈述提供了内容: 就业机会, 劳工权利, 社会保护和社会对话。 本文使第一通过其在就业政策物化这些元素的分析, 理解为一个综 合和跨国家的中央行政机关概括复杂的公共政策, 以确保促进就 业, 享有作为宪法规定的工作权利的充分机会和平等。在古巴, 就业 政策将所有有工作能力的人的保障, 自由和充分就业作为其基本原 则。然而, 在最近的立法变化和新的经济主体在法律上承认, 古巴政 府已经将这些科目策略就业政策寻求实现获得就业作为保障的要 素之一的挑战“体面劳动” 在古巴和享受“体面工作”的为有效祖国 的规则已经确立的宪法原则之一。

关键字: 体面劳动, 就业政策, 工作权, 经济主体

\section{Primeras ideas}

La contemporaneidad está caracterizada por una globalización efervescente de la que por algún tiempo se pensó que resolvería los males de la humanidad. Según Zaffaroni (2015) con la globalización se ha producido el resquebrajo de algunos derechos humanos, entre ellos uno que este mismo autor denomina "el derecho al desarrollo". La globalización ha representado el redireccionamiento de la economía hacia determinadas regiones o Estados y por ende cambios sociales importantes. Las políticas económicas globales han estado inclinadas hacia un interés neoliberal dando, nuevamente, cabida al liberalismo económico y político vulnerando las estructuras de un Estado de Bienestar General, nacido tras el fracaso de los Estados Liberales.

En el plano del trabajo fenómenos como la precarización, la flexibilización, el auge de las tecnologías, las migraciones calificadas y la discriminación laboral han puesto en riesgo 
los derechos laborales. La perspectiva sobre el trabajo había cambiado. Los logros sociales de protecciones sociales en la que intervenía el Estado como ente protector de los trabajadores, desde lo colectivo de la relación laboral, están dejando de fomentar nuevas alternativas en un empleo amplio y decente para los ciudadanos con capacidad de trabajar.

Para Sennett (2006) el trabajo comenzó a ser parte de una cadena en la que el sujeto es conducido a observarse y reconocerse desde la conformación de una temporalidad inestable y cambiante. La ausencia de contratos o contratos temporales, subcontrataciones, así como una población que no reúnen las condiciones necesarias para los requerimientos de un empleo formal, generan inseguridad, propician la aceptación de empleos sin ningún tipo de cobertura, con la flexibilidad de despido. Se violan los derechos sindicales y de negociación colectiva, por parte del empleador. Estos elementos ponen en riesgo los pilares básicos que construyen una relación laboral justa. A decir de Santamaría (2009, p. 35) la precariedad se equipará a la incertidumbre, a la inseguridad y a la falta de protección, no sólo de las condiciones de trabajo sino también de las condiciones de vida. Este fenómeno, impulsado por las necesidades económicas de los obreros y la flexibilidad de las normas jurídicas anulan o restringidos los derechos legales y beneficios sociales asociados al empleo y que se caracteriza por involucrar los siguientes tres elementos críticos: inestabilidad, ilegalidad y desprotección (Salvia y Tissera, 2002).

La flexibilización del Derecho del Trabajo elimina normas de ius cogens que otorgaban protección a los trabajadores frente a la exorbitante autonomía de la voluntad en la esfera contractual que defienden algunos empleadores. Dicha autonomía, de más decir, matizada, para los trabajadores, por la necesidad económica de solventar gastos personales y familiares que en varios lugares los obligan a aceptar condiciones precarias. Escapar de las normas jurídicas y vulnerar las regulaciones en materia de trabajo, impuesto o disposiciones migratorias, han dado auge 
sector informal. Un mercado subterráneo más precario, flexible e inestable.

Los cambios sociales y técnicos que caracterizan las recientes décadas han repercutido en el mundo del trabajo, el cual ahora se encuentra caracterizado por una mayor flexibilización, la precarización del contrato laboral y la eliminación de puestos de trabajo. En general se presenta una desproletarización del trabajo manual, industrial y fabril: heterogeneización, subproletarización y precarización del trabajo, disminución del proletariado industrial tradicional y aumento de la clase que vive del trabajo. (Quevedo, y Agudelo, 2012, p. 105).

El trabajo flexible además de generar condiciones precarias, propicia el auge de las contrataciones temporales. Arenas (2002, p. 209) entiende tres formas de contrataciones; «el monopolio en el reclutamiento de los trabajadores", conocido como la tercearización, "la flexibilidad en la terminación del vínculo» $\mathrm{y}$ "las modalidades especificas en que se presta el trabajo». La primera ha traído desprotección a la masa trabajadora pues la salida de una relación jurídica secundaria deja en manos de la primera ciertas responsabilidades desprendidas del contrato de trabajo que no se poseen con el segundo empleado. Por ello este no quedaría obligado frente a determinados sucesos como los accidentes de trabajo. Asimismo, no se desprenden obligaciones salariales ni se pueden negociar colectivamente derechos y deberes entre ambos.

En el caso de la segunda, encuentra freno en la materialización del principio de estabilidad en el empleo a través de mecanismos jurídicos que protejan de manera forzosa a los trabajadores, como es el supuesto de la defensa de la maternidad como impedimento a un despido por estado de gestación. La tercera de las manifestaciones a dado surgimiento a nuevas formas de contratos atípicos, algunos unidos al desarrollo de las tecnologías como lo es "el teletrabajo».

Esta modalidad de trabajo es una manera de organización donde el trabajador no necesita asistir a la empresa o planta 
productiva para realizar su trabajo pues lo puede llevar a cabo desde su casa en tiempo real gracias a las fuentes telemáticas. Por tanto, no necesariamente está sujeto a una jornada de trabajo pues lo importante es la entrega del resultado en el tiempo estimado. Hoy, uno de los principales problemas que giran sobre esta forma radican en el reconocimiento de iguales derechos para los trabajadores ordinarios y los llamados teletrabajadores. Derechos en cuanto a sindicación, estímulos materiales, condiciones de trabajo, riesgos laborales y otros.

Otro elemento importante que ha cobrado auge en el contexto mundial y con determinadas peculiaridades en el regional, son las migraciones. La estabilidad y seguridad de los migrantes se ha puesto en riesgo. Los países con más posibilidades económicas, han desarrollado sus economías y han recrudecido sus políticas migratorias causando vulnerabilidad a los migrantes y sus derechos.

Los trabajadores migrantes lejos están de un empleo digno y estable. De que se les respeto su "derecho al trabajo" y particularmente su "derecho a la estabilidad". Son despedidos sin justa causa y sin un derecho a reclamar en una jurisdicción social. Esto se genera por falta de políticas migratorias eficientes que protejan a los migrantes. También la falta de políticas públicas que resguarden a los trabajadores y sus familias frente a las medidas de los empleadores amparados en la exorbitancia de la autonomía para pactar cláusulas contractuales, algunas establecidas de forma verbal. El tratamiento internacional al tema ha generado, no solo Convenios de la OIT "como el 143/1975, el 111/1958 o el 97/1949" sino preocupación internacional. Tal así, en la Agenda 2030 para el Desarrollo Sostenible, la meta 8.8 hace alusión a la protección de los derechos laborales y la promoción de un entorno de trabajo seguro.

Estos fenómenos se entrelazan unos con otros y han generado para la región una pérdida de protección jurídica para los trabajadores. Si durante la revolución industrial la principal preocupación fue limitar la jornada de trabajo de manera que 
la salud de los trabajadores quedara salvaguardada, la etapa que vivimos de globalización económica y masiva utilización de nuevas tecnologías en el trabajo implica la internacionalización del empleo y la necesidad de que de las nuevas oportunidades surgidas se aprovechen debidamente, justamente, quienes prestan por cuenta ajena o propia un trabajo. (Navarro, p. 18)

La situación socioeconómica antes descrita de manera somera impulsó a la OIT a lanzar, en el año 1999, el concepto de trabajo decente. Sin embargo, válido resulta mencionar que una política de "trabajo decente», no es enarbolada por esta organización en el presente siglo, sino que forma parte de los postulados que le dieron surgimiento un centenario atrás.

La concepción del trabajo decente aparece en un momento de crisis del constitucionalismo social. Un término que, en sus inicios, nació ligado a la dignidad del ser humano. Ermida (2001, p. 13) entiende que:

el trabajo decente es un concepto integrativo en cuya realización es necesario combinar aspectos relativos a los derechos, el empleo, la protección y el diálogo en un todo integrado, agregándose que constituye una meta, un marco para la definición y actuación de políticas, un método de organización de actividades y programas, y una plataforma para la asociación con otras entidades.

Las posturas buscan enarbolar un problema actual que encierra en sí la búsqueda de soluciones a los problemas de la era globalizada. Se intenta fundamentar su postura desde la integración de todos los trabajadores y su dignidad a partir del presupuesto de la desmercantilización del trabajo. No pueden existir formas de explotación que vulneren los derechos de hombres y mujeres como seres humanos porque el trabajo es la satisfacción de las necesidades básicas para la subsistencia del trabajador y su familia.

Otro estudioso del concepto Ghai (2005), plantea que el trabajo decente debe cumplir con las siguientes características: libertad y no discriminación en cuanto a la elección del empleo, 
medidas de protección para la salud de los trabajadores, libertad de asociación y sindicalización, así como libre acceso a la negociación colectiva, la existencia de un mínimo de seguridad social y la garantía a un tripartismo y diálogo social.

Por su parte Godfrey (2003) reconoce seis dimensiones del término, donde en realidad extiende el alcance de cada una de las nociones ya identificadas. Para él (...) existe trabajo decente cuando las oportunidades para encontrar trabajo son para todos; esto incluye el autoempleo, el trabajo doméstico, y tanto el empleo asalariado en los sectores formales, e informales. El trabajo debe ser elegido en libertad, lo cual deja afuera de toda consideración al trabajo forzoso y toda forma de trabajo infantil. Debe ser productivo y justamente remunerado. Es importante el respeto a la equidad en el trabajo, incluyendo en ello la ausencia de la discriminación en el acceso, debe existir seguridad y protección social. El respeto a los trabajadores incluye poder participar, expresar libremente sus preocupaciones y reclamos; en síntesis, asociarse para poder opinar y decidir acerca de sus condiciones de trabajo.

El trabajo decente es una postura que deben asumir todos los Estado, tanto los más desarrollados como los en vías de desarrollo. Empero, son medidas que se deben aplicar sobre la base de las posibilidades económicas de cada nación. En esto juega un rol importante el reconocimiento de los derechos de los trabajadores. El propio Ghai (2005) advierte que el trabajo decente se logra si los hombre y mujeres encuentran "sus oportunidades". Las oportunidades deben ser promovidas por los Estados a través de las políticas públicas que garantice cantidad y calidad de trabajos para todos. Por tanto, la fuente del trabajo decente descansa en derechos y para que haya derechos en el trabajo lo primero es que exista trabajo, es decir empleo.

El trabajo decente es una construcción social, ética que debe informar la actuación de todos los sujetos involucrados en las relaciones de trabajo. Se puede enarbolar como un principio 
que necesita materializarse en las legislaciones de los distintos países a través del reconocimiento de derechos a los trabajadores para la consecución de sus fines. Este concepto devuelve al trabajo su dimensión social. (Weimberg, 2003) sostiene que de esta forma se permite devolverle al trabajo sus contenidos de dignidad, seguridad y humanidad, y que su incremento en la sociedad hará posible una modernidad sin exclusiones.

Es una postura integrativa que no puede verse solo a partir de un principio. No existe trabajo decente sin el engranaje de estos elementos, pero sobre todo sin políticas públicas de acceso al empleo eficientes, integrativas e inclusivas. El punto de partida está en la posibilidad de empleos. Posibilidad que subyace en manos de las políticas públicas de empleo de los gobiernos como primer eslabón. Luego, en la garantía de una estabilidad laboral para poder dar cometido al resto de las cuestiones que garantizan la decencia del trabajo contra la precariedad a la que se ven sometidos los trabajadores en el siglo XXI, donde intervienen los empleadores, sindicatos y organizaciones internacionales. Por ende, en este ensayo se entiende que el trabajo decente no es un concepto jurídico, sino una definición de carácter social que necesita, no solo de mecanismos jurídicos para su desarrollo sino de la intervención de una institucionalidad que lleve a vías de hecho los derechos que la norma establece en materia de trabajo y protección social. Para hablar de trabajo decente se necesitan: políticas públicas de acceso al empleo, derechos laborales, protección social y diálogo social tripartito.

\section{Apuntes sobre la Política de empleo}

Las políticas de empleo son políticas públicas en manos de los órganos de la Administración Pública para garantizar el "derecho al trabajo" de los ciudadanos de un Estado y el pleno desarrollo de este a partir de la importancia social del trabajo para la satisfacción de necesidades personales y familiares de los trabajadores. La Organización de Cooperación y Desarrollo 
Económico (OCDE) formalizó la clasificación de las políticas públicas en pasivas y activas.

Neffa (2011) refiere que son políticas activas cuando contienen un objeto triple, primeramente, cuando buscan reducir el desempleo, además buscan modificar el nivel y la calidad de la fuerza de trabajo, para así satisfacer las demandas de empleo por parte de los trabajadores y como tercer elemento se constituyen además por el cambio de la naturaleza de la demanda, es decir, buscan el aumento de la cantidad y la calidad de las ofertas de empleo que hacen los trabajadores. A su vez se denominan políticas pasivas las que buscan garantizar un mínimo de recursos con el objetivo de asegurar la reproducción de la fuerza de trabajo, dirigidas a la fuerza de trabajo más vulnerable, es decir, a las personas sin empleo. La medida pasiva más importante consiste en otorgar a los desocupados un subsidio por desempleo o en su defecto, otorgar los beneficios de políticas sociales u otorgar una asistencia de contenido alimentario.

Esta diferenciación entre políticas activas y pasivas no es excluyente, puesto que pueden complementarse o, simplemente, lo que comenzó siendo una política activa termina transformándose en una pasiva.

La política de empleo se basa en la estrategia que debe desarrollar un país para propiciar el crecimiento de los empleos dignos y productivos para todas aquellas personas que formen la fuerza laboral. Estas estrategias deben evitar el desempleo y fomentar la estabilidad en el empleo, mediante la coordinación con las políticas en materia económica y social y su integración a la planificación. Por ellos, las políticas de empleo se alzan como uno de los mecanismos con que cuenta el Estado para mantener el equilibrio en la balanza entre la fuerza de trabajo y la capacidad de empleos. Es una forma eficiente de hacer frente a los problemas de desempleo y estabilidad en el empleo, clásicos en muchos sistemas capitalistas de hoy.

Las políticas públicas de empleo, si bien se conciben como responsabilidad de los órganos del Estado, responsabilizados 
con las temáticas laborales, debe entenderse en un sentido transversal. Hoy en día, se hace imposible concebir que las estrategias de políticas públicas queden solamente en mano de un solo órgano. La tranversalización es un elemento clave en una labor gubernativa eficiente. Por ello en materia de empleo, donde intervienen instituciones afines a la educación general o universitaria, la salud pública, la cultura, el deporte etc. resulta útil que todos asuman determinas funciones en ello. Funciones que pueden estar encaminadas no solo al fomento de puestos de trabajos, sino a la equidad de género, la no discriminación, la superación y capacitación profesional entre otros elementos que permitan eliminar las trabas para que los ciudadanos accedan a empleos.

Desde la Organización Internacional del Trabajo se brinda apoyo para formular y poner en marcha políticas de empleos a niveles nacionales, buscando la correspondencia entre crecimiento económico, políticas económicas y la creación de empleos. Para ello hace un estudio conjunto de las oportunidades y zonas favorables a la hora de generar trabajo productivo. A través de sus Convenios ${ }^{2}$ esta organización ha llamado a políticas de empleo que garanticen empleos dignos para todos. El Plan de Acción 2010-2016 (OIT, 2011) expone:

Estos convenios desempeñan una función esencial en la promoción de un empleo pleno, productivo y libremente elegido, en el fortalecimiento de la cohesión social por medio del diálogo social, y en el mantenimiento de las condiciones decentes de trabajo por medio de un servicio de inspección del trabajo eficaz. Contribuyen a allanar el camino para salir de la crisis actual. (p.1)

2 Entre los Convenios de este organismo internacional resaltan: el Convenio relacionado con el fomento del empleo y protección contra el desempleo (OIT, 1988); el Convenio sobre trabajo forzoso (OIT, 1930); Convenio sobre el servicio de empleo (OIT, 1948) y el Convenio sobre la discriminación (empleo y ocupación) (OIT, 1958), el Convenio sobre la política de empleo (OIT, 1964) 
Por ende, sobre estos postulados el Estado cubano ha esbozados su política de empleo, la cual está llamada a cambios importantes de cara el nuevo contextos económico y laboral.

\section{La política de empleo en Cuba y la inclusión de los nuevos actores económicos}

El triunfo de la Revolución cubana en enero de 1959 trajo grandes cambios para el país en todas las esferas. A pesar que, desde la Constitución de 1940, se recogió el derecho al trabajo y otra gama de derechos constitucionales ${ }^{3}$ afines al trabajo, no fue hasta la década de 1960 que comenzaron cambios significativos más allá de su enunciación jurídica. La construcción de una sociedad socialista levantada de la mano de la clase obrera, generó la protección de la masa trabajadora.

El Estado cubano se construyó sobre la base de la propiedad estatal y social de los medios de producción. A partir de las nacionalizaciones, permitió un mejor control de los recursos laborales a partir de políticas concretas, como la del empleo, en un contexto que heredaba una alta descapitalización económica (González e Izquierdo, 2016). Por tanto, durante casi medio siglo, desde 1960 hasta iniciado el siglo XXI, el Estado era el único y principal empleador, lo que permitía una "política de pleno empleo» basada en la libertad para elegir trabajo y la igualdad de oportunidades, construyendo las relaciones de trabajo sobre el principio rector de idoneidad demostrada (Código de Trabajo, 2014, Art. 36).

De igual manera la política socio laboral cubana también se apoyaba en el desarrollo educacional de las políticas estratégicas de desarrollo social que buscaban reconfigurar la estructura de las fuerzas de trabajo y el progreso de los territorios más desfavorecidos (Izquierdo Quintana, 2017, p. 120).

\footnotetext{
3 Vid. Artículos 43 y sig., 60 y ss. Ley de 1 ro de julio de 1940 (Constitución de la República de Cuba) en Gaceta Oficial No. 464 de fecha 8 de julio de 1940.
} 
Sobre el tratamiento a la temática en estos primeros años en Cuba autores patrios como Viamontes (2001) expresan:

Es en estos primeros años que surge la política de empleo centralizada, cuyo signo característico fue la distribución y redistribución de la fuerza de trabajo por parte de organismos gubernamentales teniendo en cuenta fundamentalmente las necesidades económicas del país para evitar un desbalance en la ocupación de la fuerza de trabajo. Si bien este sistema fue la solución más acertada para aquel momento, debe referirse que los elementos relativos a motivaciones específicas de los trabajadores $o$ de una entidad laboral determinada en el plano de relaciones laborales no eran reconocidos legalmente. (p. 105)

La política de empleo impulsada por un principio de centralización necesitó de asiento legislativo. Por ello se generaron regulaciones que garantizaran oficios para todos, bajo la base de la no discriminación. Así existen en el ordenamiento laboral protecciones a sectores vulnerables como los discapacitados, los licenciados del servicio militar activo, o los que cumplen sanción penal (Código de Trabajo, 2014, Art. 4). De igual manera un tratamiento diferente para los jóvenes entre 15 y 16 años (Código de Trabajo, 2014, Art. 64 al 68) que excepcionalmente pueden incorporarse a trabajar antes de la edad establecida de 17 años (Código de Trabajo, 2014, Art. 22). Una protección a este sector, concatenada con la obligatoriedad de estudiar, establecida desde la norma constitucional (Const, 2019, Art. 73) y el sistema de educación gratuito y público, que permite, no solo alcanzar nivel educaciones superiores sino adquirir los niveles técnicos y habilidades necesarias para incorporarse a determinadas profesiones $\mathrm{u}$ oficios. El rector de estas políticas es el Ministerio de Trabajo y Seguridad Social (MTSS), titular principal de la Política de empleo, quien puede asignar la contratación de estas personas por interés social y estatal (Código de Trabajo, 2014, Art. 21).

Otro elemento de garantía al empleo en el ordenamiento jurídico patrio, y que repercute en un hecho social importante 
en el país es el Servicio Social para los jóvenes que egresan de la enseñanza técnica y profesional y los graduados de estudios universitarios de pregrado. El servicio social en el terreno nacional está estipulado (Código de Trabajo, 2014, Artículo 69 y sgts) como un deber de los sujetos que terminan los niveles de enseñanza mencionados con el objetivo de que adquieran las habilidades técnicas para desempeñarse en los puestos de trabajo. Ello constituye también un mecanismo de garantía de empleo que se mantendrá, una vez culminado los períodos establecidos para el cumplimiento de dicho servicio " 2 años los hombres y 3 las mujeres" si demuestran poseer la idoneidad demostrada ${ }^{4}$ para el puesto de trabajo.

El servicio social por ende posee una doble finalidad. Primeramente, para protegerlos por la nula o poca experiencia con que cuentan respecto a la fuerza laboral predominante. En segundo lugar, para responder de las necesidades que presenta la sociedad y la economía nacional. Nuestro Código de Trabajo en su artículo 69 (Código de Trabajo, 2014) formula que este cumplimiento además va a ser consecuente con la planificación y las prioridades del desarrollo económico y social. Esto como se ha mencionado posee características coherentes con la forma de económica planificada del Estado cubano que posibilita garantizar el acceso a empleos generando su creación (Anuario Estadístico de Cuba, 2018) ${ }^{5}$ para los ciudadanos cubanos con capacidad de trabajar.

\footnotetext{
4 La idoneidad demostrada es el principio rector de la contratación laboral en Cuba. Constituye el elemento por el cual puede ser terminada la relación jurídica laboral por iniciativa del empleador, cumpliendo las garantías establecidas en la propia norma nacional para determinar la perdida de la idoneidad. Es un mecanismo de garantía de la política de empleo y del derecho a la estabilidad en el trabajo. Vid. Artículo 36 de la Ley 116/2013. Publicado en la Gaceta Oficial de la República No. 29 Extraordinaria de 17 de junio de 2014 y Artículos 22 al 29 del Decreto 326/2013. Reglamento del Código de Trabajo. Publicado en la Gaceta Oficial de la República No. 29 Extraordinaria de 17 de junio de 2014

5 El Anuario Estadístico de Cuba 2018 publicó que la, la tasa de desempleo al cierre del 2017 era de 1,7 por ciento. Disponible en: http://www.one.cu/aec2017.htm el 23 de abril de 2019
} 
Sin embargo, la centralización de la política de empleo como principio, es relativo. No queda limitada o restringida en la normativa nacional la posibilidad de que los empleadores (Código de Trabajo, 2014, Art. 21) contraten fuerza de trabajo o seleccionen del trabajador que mejor posea determinada capacidad jurídica para un puesto de trabajo. Fue desde el Decreto 73 (1980) que se empieza a llevar a cabo la descentralización de la política de empleo, pero bajo la garantía de reservar determinados puestos de trabajo para los trabajadores por interés social o estatal, tal como se mencionó con anterioridad.

Otro elemento de la Política de empleo y que ha nacido de la iniciativa de la Organización Internacional del Trabajo (OIT), ha sido la formación profesional de los trabajadores como elemento integrante de la política de empleo. Ermida (2001) expresa que:

(...) la formación profesional es un derecho humano fundamental que en tanto tal se impone por sí mismo entre aquellos derechos que deben ser respetados en todo trabajo decente; pero al mismo tiempo es un instrumento que facilita y a veces condiciona la realización de otros derechos que también constituyen el trabajo decente. (p. 17)

La superación y capacitación en Cuba ha sido considerada como uno de los derechos de los trabajadores. Así queda regulada en la norma nacional (Código de Trabajo, 2014, Art. 2 e)). De igual manera se reconoce la obligación de los trabajadores de elaborar anualmente los planes de capacitación de los trabajadores, así como capacitar a los trabajadores de nuevo ingreso (Código de Trabajo, 2014, Art. 39).

Desde el 2010, en el VI Congreso del Partido Comunista de Cuba fueron aprobados los Lineamientos de la Política Económica y Social del Partido y la Revolución (PCC, 2011). En uno de los lineamientos se reconoce la necesidad de la apertura al sector no estatal como complemento necesario en la economía nacional. A partir de ello, se comenzó a regular en el país del 
denominado "trabajo por cuenta propia", generando una nueva forma de empleo que hoy representa el $12 \%$ de la fuerza de trabajo. En el contexto cubano existen hoy nuevos actores económicos, algunos reconocidos desde las modificaciones a la ya derogada Constitución de 1976, tras la reforma de 1992. En este supuesto se encuentran los sujetos económicos de la inversión extranjera (Ley 118, 2014) que pueden ser empresas de capital totalmente extranjero y de capital mixto. Fue, a partir de la última década que junto al trabajo por cuenta propia entran en el contexto nacional las cooperativas no agropecuarias (DecretoLey 305, 2012), pues las cooperativas agropecuarias fueron reguladas en Cuba desde inicios de la Revolución.

Estos actores económicos han quedado reconocidos en la Constitución de la República de 2019 en su artículo 22 (Const., 2019). En el mismo se reconocen junto a la propiedad «socialista de todo el pueblo" como forma principal "la cooperativa» "mixta» "privada" "de instituciones y formas asociativas» $\mathrm{y}$ "personal». Algunas de ellas pueden dar lugar a nuevos sujetos económicos que hoy operan en el sector económico cubano o contratan fuerza de trabajo. Por tanto, necesitan involucrarse en los mecanismos de política de empleo, que hasta el momento están destinada solo al sector empresarial estatal, como sujeto principal. De cara a este contexto urge incorporar a los mecanismos de política de empleo estos nuevos sujetos sobre la base de los propios fundamentos económicos del Estado cubano.

La propia Constitución de 2019 establece, en cuanto a la política de empleo, que el Estado posee dentro de los postulados normativos dedicados a los «Fundamentos de la Política Educacional, Científica y Cultura" que uno de sus principios será el fomentó a la formación y empleo de las personas que el desarrollo del país requiere (Const. 2019, Art. 32 g)).

A partir de aquí los órganos de la Administración Pública cubana poseen retos importantes de cara a estos nuevos sujetos económicos. A pesar de estos mecanismos de pleno empleo, el reciente surgimiento de empleadores no estatales producto de 
la apertura al trabajo por cuenta propia en la isla acarrea desafíos para la incorporación de este sector a las políticas de empleo que le permitan convertirse en sujetos empleadores responsables ante sectores vulnerables. También posibilitaría poder acceder a la fuerza de trabajo que termina en los distintos niveles de enseñanza a través, por ejemplo, de la asignación de jóvenes que cumplen el servicio social. De esta forma se disminuiría la desprofesionalización de los oficios al no acceder a las personas tituladas y calificadas para determinadas laborales y crear una sinergia con todos los sujetos que emplean.

De igual manera en el país existen las oficinas de las Direcciones Municipales de Trabajo y Seguridad Social, subordinadas a las Asambleas Municipales del Poder Popular y metodológicamente al Ministerio de Trabajo y Seguridad Social. En ellas existe la disponibilidad de puestos de trabajo en el territorio perteneciente al municipio al que se pueden dirigir las personas que desean buscar empleos. Esta es otro punto que necesita una redefinición. Las convocatorias a puestos de trabajo en el sector no estatal no se encuentran disponibles en estos lugares. Hoy la Constitución cubana llama a la autonomía municipal. Por ende, estos órganos del Poder Popular pueden generar mecanismos efectivos que logren incorporar, desde la labor de los municipios, a los sujetos de gestión de estatal a esta forma de divulgación de los empleos.

En cuanto a la formación y capacitación como mecanismo de la política de empleo también se necesita, en primer lugar, la concientización por parta de los empleadores privados de que la formación profesional constituye un derecho fundamental de los trabajadores. a partir de aquí la generación de presupuestos para cubrir los gastos de superación y capacitación de los trabajadores. en este punto también juega un papel la iniciativa de los gobiernos municipales, junto a ello una labor concatenada entre los Ministerio de Trabajo y Seguridad Social (MTSS) y Educación Superior (MES) y Educación (MINED) generando los mecanismos que permitan la creación de esto espacios y que atiendan a las necesidades de sector laboral. 
En materia de orientación e identidad sexual aún son tarea pendiente en la normativa laboral cubana, aunque tras la entrada en vigor de la reciente Constitución de 2019 se avizora un camino certero (Constitución, 2019, Artículo 40 y 42). En este supuesto será importante que los destinatarios de las políticas de genero vinculadas al acceso al empleo estén destinados para todos los sujetos económicos que poseen la facultad de contratar fuerza de trabajo.

\section{Conclusiones}

La política de empleo en Cuba ha estado caracteriza por una política de pleno empleo e igualdad de todos los sujetos en capacidad de trabajar. Sin embargo, ha estado y continúa estando construida de cara al sector empresarial estatal. Las políticas públicas de acceso al empleo están llamadas a perfeccionar mecanismos jurídicos, adaptarlos a los nuevos actores económicos, acoplar los nuevos derechos que se traen a relucir en el contexto jurídico, a cambiar las mentalidades y romper estereotipos innecesarios.

El aporte fundamental de la política socio laboral cubana a una concepción del trabajo decente surge de la comprensión institucional de que su materialización se realiza en un sistema de relaciones en y para el trabajo al ir más allá de lo meramente económico, como muy recurrentemente se ha querido demostrar (Izquierdo Quintana, 2017, p. 129).

La concepción de trabajo decente es un todo engrando que necesita, de políticas públicas que garanticen el empleo para todos los ciudadanos en capacidad de trabajar, sin ningún tipo de discriminación. La cuestión no radica solo en la generación de empleos, sino en mecanismos que aseguren en acceso de todos en dependencia de sus condiciones particulares. De ahí la necesidad de incorporar a todos los trabajadores como garantía también del principio protectorio del Derecho del Trabajo. Asimismo, un trabajo decente será posible no solo por generar nuevos empleos, sino que estos tengan la calidad necesaria. 
Una calidad entendida como el andamiaje jurídico que permita un efectivo disfrute de todos los derechos laborales.

Cuba tiene un camino por delante para adaptarse a cambios importantes es su estructura socioeconómica, pero lo cierto es que siempre ha apostado, desde las normas jurídicas por proteger a los trabajadores. Las políticas de empleo han buscado la garantía de puestos de trabajo como primer eslabón para el disfrute de los derechos laborales y demás derechos en el trabajo.

\section{Referentes Bibliográficos}

Ermida Uriarte, O. (2001) Trabajo decente y formación profesional. Boletín interno 151, CINTERFOR, Montevideo.

Ghai, D. (2002). Decent work: concepts, models and indicators. IILS, DP/139/2002. Suiza.

Ghai, D. (2005). Decent work: universality and diversity. IILS, DP/159/2005, Suiza.

Godfrey, M. (2003). Employment dimensions of decent work: tradeoffs and complementarities. IILS, DP/148/2003, Suiza.

Gómez, V (2010). El diseño de las políticas activas de empleo en un nuevo contexto de crisis. Una reflexión. España. La Ley.

Izquierdo Quintana, Osnaide; Burchardt, Hans-Jurgen (2017). Trabajo decente y Sociedad. Cuba bajo la óptica de los estudios socio laborales. Editorial UH. La Habana.

Neffa, J. C (2011). Empleo, desempleo y políticas de empleo. Recuperado de http//www.ilo.org/

Organización Internacional del Trabajo (1999), Trabajo decente, Memoria del Director General a la $87^{\mathrm{a}}$ reunión de la Conferencia Internacional del Trabajo, Ginebra.

Santamaría López, Elsa. (2009) Reseña crítica: Precariedad laboral: apuntes para una aproximación sociológica a sus formas contemporáneas, en Papeles del CEIC (Revisión Crítica), vol. 1, $\mathrm{n}^{\circ}$ 6, CEIC (Centro de Estudios sobre la Identidad Colectiva), Universidad del País Vasco, Disponible en: http://www.ehu. es/CEIC/pdf/critica6.pdf 
Vejar Dasten Julián. (2014) La precariedad laboral, modernidad y modernización capitalista: Una contribución al debate desde América Latina. Revista Trabajo y Sociedad. No. 23 Invierno. Argentina, Disponible en: www.unse.edu.ar/trabajoysociedad

\section{Legislación Nacional}

Ley de 1ro de julio de 1940 (Constitución de la República de Cuba) en Gaceta Oficial No. 464 de fecha 8 de julio de 1940

Constitución de la República de Cuba de 2019 en Gaceta Oficial Extraordinaria No. 5 de 10 de abril de 2019.

Ley 116/2014 "Código de Trabajo" en Gaceta Oficial Extraordinaria No. 29 de 17 de junio de 2014

Decreto Ley 339 "De la maternidad de la trabajadora". en Gaceta Oficial Extraordinaria No.7 de 10 de febrero de 2017 de la República de Cuba

\section{Normas Internacionales}

Organización Internacional del Trabajo (1964), Convenio sobre politica de empleo. http// www.ilo.org/

Organización Internacional del Trabajo (1988), Convenio sobre el fomento al empleo y protección contra el desempleo. http// www.ilo.org/

Organización Internacional del Trabajo (1948), Convenio sobre el servicio del empleo. http// www.ilo.org/

Organización Internacional del Trabajo (1964) Recomendación sobre la politica de empleo. http// www.ilo.org/

Organización Internacional del Trabajo (2011). Plan de Acción 2010-2016. Recuperado de http// www.ilo.org/ 